\title{
BMJ Open A study of the nature and level of trust between patients and healthcare providers, its dimensions and determinants: a scoping review protocol
}

\author{
Supathiratheavy Rasiah (10 , ${ }^{1}$ Safurah Jaafar, ${ }^{1}$ Safiah Yusof, ${ }^{2}$ \\ Gnanajothy Ponnudurai, ${ }^{3}$ Katrina Pooi Yin Chung, ${ }^{4}$ Sasikala Devi Amirthalingam ${ }^{5}$
}

To cite: Rasiah S, Jaafar S, Yusof S, et al. A study of the nature and level of trust between patients and healthcare providers, its dimensions and determinants: a scoping review protocol. BMJ Open 2020;10:e028061. doi:10.1136/ bmjopen-2018-028061

- Prepublication history for this paper is available online. To view these files, please visit the journal online (http://dx.doi org/10.1136/bmjopen-2018028061).

Received 08 May 2019 Revised 13 November 2019 Accepted 14 November 2019

Check for updates

(C) Author(s) (or their employer(s)) 2020. Re-use permitted under CC BY-NC. No commercial re-use. See rights and permissions. Published by BMJ.

${ }^{1}$ Community Medicine, International Medical University, Bukit Jalil, Kuala Lumpur, Malaysia

${ }^{2}$ Nutrition and Dietetics, International Medical University, Bukit Jalil, Kuala Lumpur,

Malaysia

${ }^{3}$ Human Biology, International Medical University, Bukit Jalil,

Kuala Lumpur, Malaysia

${ }^{4}$ Pathology, International Medical University, Bukit Jalil, Kuala

Lumpur, Malaysia

${ }^{5}$ Family Medicine, International Medical University, Bukit Jalil, Kuala Lumpur, Malaysia

Correspondence to Dr Supathiratheavy Rasiah; supathiratheavy@imu.edu.my

\section{ABSTRACT}

Introduction The aim of this scoping review is to systematically search the literature to identify the nature and or level of trust between the patient, the users of health services (eg, clients seeking health promotion and preventive healthcare services) and the individual healthcare providers (doctors, nurses and physiotherapists/ occupational therapists), across public and private healthcare sectors, at all levels of care from primary through secondary to tertiary care. It also aims to identify the factors that influence trust between patients, users of health services (clients) and providers of healthcare at all levels of care from primary care to tertiary care, and across all health sectors (public and private). The study will also identify the tools used to measure trust in the healthcare provider.

Methods and analysis The scoping review will be conducted based on the methodology developed by Arksey and 0'Malley's scoping review methodology, and Levac et al's methodological enhancement. An experienced information specialist (HM) searched the following databases MEDLINE, EMBASE, the Cochrane Library, Cumulative Index to Nursing and Allied Health Literature. The search terms were both keywords in the title and/or abstract and subject headings (eg, MeSH, EMTREE) as appropriate. Search results were downloaded, imported and stored into a 'Refworks' folder specifically created for reference management. The preliminary search was conducted between 7 December 2017 and 14 December 2017. Quantitative methods using content analysis will be used to categorise study findings on factors associated with trust between patients, clients and healthcare providers. The collection of studies will be also examined for heterogeneity. Qualitative analysis on peer reviewed articles of qualitative interviews and focus group discussion will be conducted; it allows clear identification of themes arising from the data, facilitating prioritisation, higher order abstraction and theory development. A consultation exercise with stakeholders may be incorporated as a knowledge translation component of the scoping study methodology.

Ethics and dissemination Ethical approval will be obtained for the research project from the Institutional Review Board. The International Medical University will use the findings of this scoping review research to improve the understanding of trust in healthcare, in its endeavour
Strengths and limitations of this study

- This study seeks to identify the level and nature of trust in healthcare between patients, users of health services and specific individual healthcare providers, for example, physicians, surgeons, nurses, community health workers, physiotherapists and occupational therapists, and pharmacists.

- It will review the literature across all levels of care from primary care to tertiary care, and in the private and public sector

- It also seeks to identify the factors that influence trust and the tools used to measure trust in healthcare providers.

- The study reviews articles published only in English and over a period of 10 years between January 2007 and December 2017.

- The scoping review will not include trust in the provision of health services by dentists, allied health professionals such as phlebotomists, medical laboratory scientists, dietitians and social workers, and in the area of mental health, and trust at the macro level or health systems level, so as to be focused in the scope covered.

to improve health services delivery in its healthcare clinics and hospitals, and in its teaching and learning curriculum. The findings will also help faculty make evidence based decisions to focus resources and research as well as help to advance the science in this area. Dissemination of the results of the scoping review will be made through peerreviewed publications, research reports and presentations at conferences and seminars.

\section{INTRODUCTION}

\section{Context of healthcare provision}

The provision of healthcare occurs in a setting characterised by uncertainty and an element of risk as to the competence and intentions of the healthcare providers. ${ }^{1}$ Traditionally, it has been widely accepted that the users or consumers of the service (ie, the patients, and the clients who come for health promotion 
and preventive healthcare services) trust the judgement, knowledge and expertise of the health professional to provide a competent service. ${ }^{2}$ The effective delivery of healthcare requires both the supply of healthcare as well as the acceptance and use of services by the patient and clients. Patient-provider interaction is at the heart of healthcare provision. ${ }^{2}$ The nature and environment of healthcare provision occurs on a relational basis-relationships between the providers and users of the service which consequentially impact on health outcomes and wellness.

\section{Trust and its importance in healthcare}

Trust is a relational notion between people, people and organisations, and people and events. ${ }^{3}$ Patient's trust in the physician can be defined as a collection of expectations that the patients have from their doctor. ${ }^{4}$ It can also be defined as a feeling of reassurance or confidence in the doctor. ${ }^{5}$ It is an unwritten agreement between two or more parties for each party to perform a set of agreed upon activities without 'fear of change from any party'. ${ }^{6}$ This is especially true in relationships that result from a lack of choice or occur in a context of asymmetry, such as that between the healthcare provider and patient. Thus, trust is a set of expectations that the healthcare provider will do the best for the patient, and with good will, recognising the patient's vulnerability. Trust facilitates cooperation between people (known to each other and/or strangers) that is catalysed, facilitated and sustained by trust. ${ }^{7}$ Trust is fundamental to effective interpersonal relations and community living. ${ }^{7}$ It forms a fundamental basis in the provision of healthcare.

Trust between the patient and the healthcare provider (doctors, nurses, physiotherapists/occupational therapists) is important in provider-patient interaction and rapport. It influences patient management outcomes, especially in the treatment of long term illness, as well as influences outcomes of health promotion and prevention initiatives. A trusting relationship between healthcare provider and patient can have a direct therapeutic effect. ${ }^{8}$ Trust relations can be distinguished at the micro and macro levels. At the micro level, Trust can be interpersonal trust which is that trust between the individual patient or individual client and the individual clinician, or between two clinicians; organisational or institutional trust is that between the clinician and the manager of the organisation. Trust at the macro level includes trust between patients, the public and the organisation or institution. This study will focus on interpersonal trust between the patient or client and the individual healthcare provider.

Trust is typically associated with high quality communication and interaction, which facilitates disclosure by the patient, enables the practitioner to encourage necessary behaviour changes and may permit the patient greater autonomy in decision-making about treatment. ${ }^{9}$

Understanding the issues that influence a person's trust in the healthcare provider will assist in drawing up suitable operational policies in the delivery of healthcare, as well as influence healthcare practices and behaviours among providers. Transferring this knowledge to medical education will create an emerging practitioner who will be more aligned to the patients' needs.

\section{Erosion of trust in health care}

Critical incidents and sentinel events have contributed to erosion of the patients' trust in healthcare, the institutions and health systems. ${ }^{10}$ The changing sociopolitical environment in healthcare, the impact of the era of information technology and the fact that patients have become increasingly empowered to make informed decisions, have influenced the nature of trust in the healthcare provider. ${ }^{11}$

The aim of this scoping review is

1. To systematically search the literature to identify the nature and or level of trust between the patient, the users of health services and the individual healthcare providers, across public and private healthcare sectors, at all levels of care from primary through secondary to tertiary care.

2. To identify the factors that influence trust between patients and healthcare providers, at all levels of care from primary through to tertiary level of care, and across all sectors-public and private.

3. To identify the tools used to measure trust in healthcare between patients, clients and providers of healthcare.

\section{Conceptual framework}

Figure 1 depicts the conceptual framework for trust in healthcare. The study will explore the nature and or the level of trust at the micro-level between patients and users of health services and the individual healthcare provider. The study will also explore the factors that influence trustbetween patients and healthcare providers.

\section{METHODS}

\section{Commissioning agency}

This study is commissioned by the International Medical University, Kuala Lumpur Malaysia. The university has identified research on 'Trust in Healthcare' as one of its research thrust areas in its journey towards becoming the centre for research on trust in healthcare.

\section{Study design}

The scoping review will be conducted based on the methodology developed by Arksey and O'Malley's ${ }^{12}$ scoping review methodology, and Levac et al $\mathrm{s}^{13}$ methodological enhancement. This framework identifies six stages in undertaking a scoping review: (1) identifying the research question, (2) identifying relevant studies, (3) selecting studies, (4) charting the data, (5) collating, summarising and reporting the results. The Preferred Reporting Items for Systematic Reviews and Meta-Analyses (PRISMA) checklist and the PRISMA 2009 flow diagram will be used as a checklist in designing, reviewing and reporting this scoping review. 


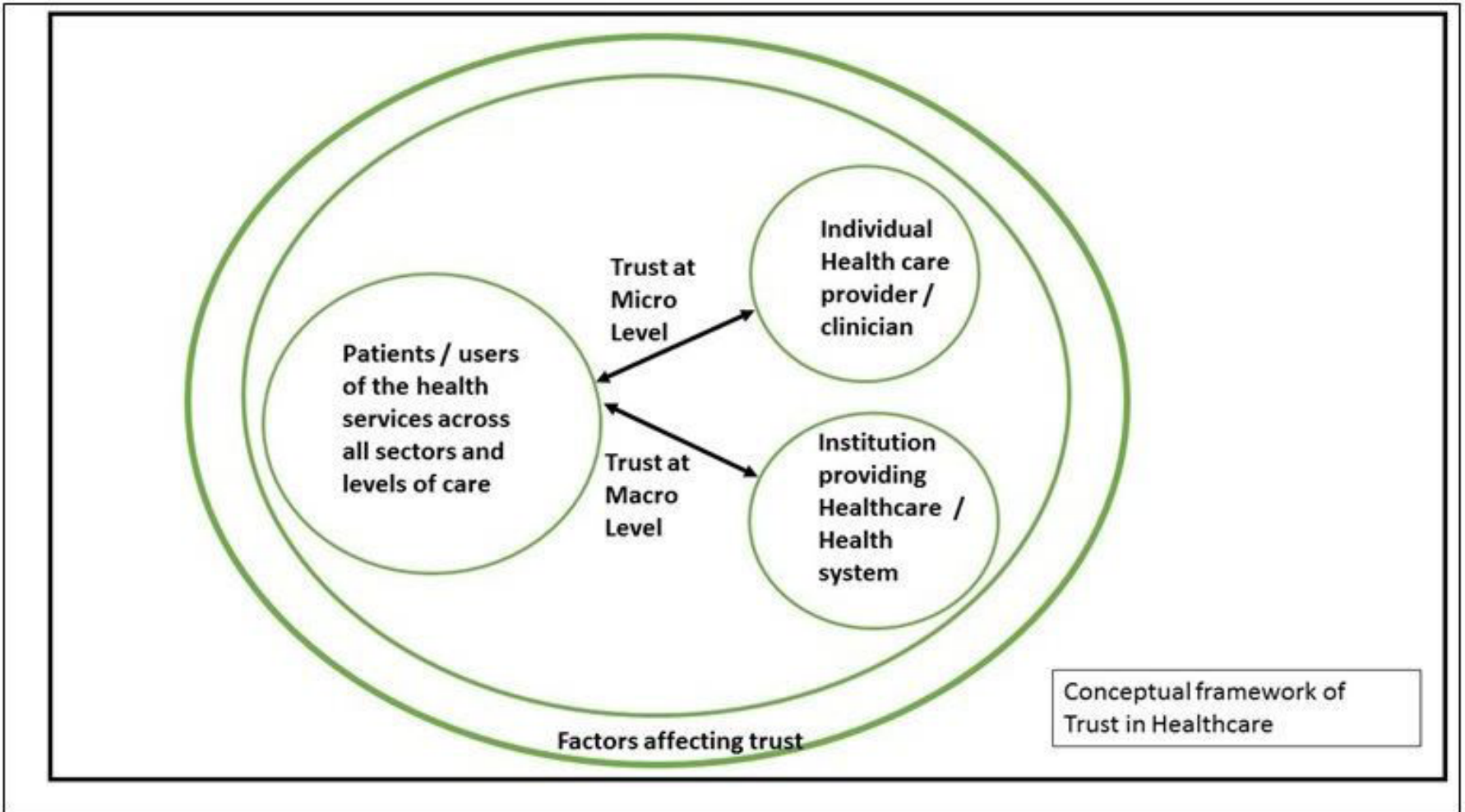

Figure 1 Conceptual framework of trust in healthcare.

\section{Stage 1: identifying the research question}

The research questions are:

1. What is the nature and or level of trust between the patient, the users of health services (clients) and the individual healthcare providers (interpersonal trust) across public and private healthcare sectors, at all levels of care from primary through secondary to tertiary care?

2. What are the factors that influence trust between patients, users of health services and providers of healthcare?

3. What are the tools used to measure trust in healthcare at the interpersonal level?

\section{Stage 2: identifying relevant studies}

The scoping review will be as comprehensive as possible in identifying primary studies and reviews answering the research questions. The research will be restricted to publications in English between the time period of January 2007 and December 2017 and adhere to the eligibility criteria. A preliminary search was conducted between 7 December 2017 and 14 December 2017.

\section{Information sources and search strategy}

An experienced information specialist (HM) searched the following databases MEDLINE, EMBASE, the Cochrane Library, Cumulative Index to Nursing and Allied Health Literature. The search terms were both keywords in the title and or abstract and subject headings (eg, MeSH, EMTREE) as appropriate. Search results were downloaded and imported and stored into a 'Refworks' folder specifically created for reference management. The preliminary search was conducted between 7 December 2017 and 14 December 2017.

A variety of grey literature will also be searched through the websites of relevant agencies such as the National Institutes of Health, National Institute for Health and Care Excellence and Agency for Healthcare Research and Quality, to identify studies, reports and conference abstracts of relevance to the research questions of this review. We will also conduct a targeted search of the grey literature in local, provincial, national and international organisations' websites and related health or scientific organisations. Supplementary articles may be obtained by contacting field experts and searching references of relevant articles.

\section{Stage 3: study selection}

Study selection process

First step: Study selection will be initiated using screening procedures to pull together only potentially eligible studies for the scoping review. It involves two steps of screening. The first step will be to go through all the collected titles and abstracts by two independent reviewers. All retrieved citations are subjected to a set of minimum inclusion criteria. These criteria were tested a priori on a sample of abstracts to ensure that they are robust to capture articles that may relate to 'Nature and Levels of Trust in Healthcare providers'. Any discrepancies will be resolved either through consensus or, if needed, involvement of a third reviewer. Finally, articles that are selected as deemed relevant by either or both of the reviewers will be included in the full-text review in the second step screening. The 
online or e-learning articles are not included in the study selection for inclusion.

In the second step, both the reviewers will be assigned to the same articles and assess them in full text. Any disagreement between the reviewers will be resolved through discussion with a third reviewer, and thus facilitating consensus for final inclusion. An inter-rater reliability calculation may be done if needed.

\section{Eligibility criteria}

Titles and abstracts of articles which directly matched the identified keywords from year 2007 to 2017 will be filtered for relevance to nature and level of trust between healthcare providers and patients or users of health services. We will include studies that fulfil the following criteria:

1. The study reported qualitative and or quantitative data on the nature of trust or levels of trust between healthcare providers and patients or users of health services.

2. The study took place in a healthcare setting.

3. The study was published or reported in the English language.

4. The study was published in journals, reports or in conference proceedings as literature.

5. The study measured interpersonal trust (eg, trust in the nurse, physician, healthcare provider) with a valid, reliable instrument and used an established trust questionnaire (ie, included a reference to a published article which used the respective trust questionnaire) or used a validated questionnaire.

6. The study looked at factors affecting trust in healthcare between patients, clients and the healthcare provider.

7. Studies using unvalidated instruments, single item questionnaires or those measuring trust in non-health related environment will be excluded.

\section{Stage 4: data collection}

Data items and data abstraction process

A data extraction form will be created by the research team. This form will be reviewed and pretested by all reviewers before implementation to ensure that it captures the information accurately. All reviewers will be trained and be given an exercise using a random sample of articles to be included in the study. The data extraction form will also be piloted on a sample of five articles by the reviewers involved in the scoping study. The aim is to assess for completeness and ease of use. The percentage of agreement between reviewers will also be measured with a target of at least 80 percent agreement.

To ensure study relevance, the various study characteristics are listed below and, this includes but is not limited to the following:

1. Author.

2. Publication year.

3. Source origin/country of origin.

4. Aims/purpose of the study.

5. Research/study design.

6. Methodology.
7. Population characteristics (eg, number of participants, country, physician specialty).

8. Nature of Healthcare settings-hospital, clinic types, unit/department, primary care/secondary care/tertiary care, public or private sector.

9. Description of quality indicators including definition, numerator, dominator, psychometrics of the indicators (face validity, reliability, construct validity, risk adjustment).

10. Intervention characteristics (eg, concept, duration, engagement strategy, timing, required resources).

11. Tools used to measure level of trust, physician engagement, intervention results (eg, barriers, facilitators, outcomes).

12. Any factors reported to be associated with hospital physician engagement:

- Demographics.

- Characteristics of the work environment (eg, organisational support, quality of work-life and perceptions of safety).

- Work attitudes (eg, physician work engagement, job satisfaction, commitment and empowerment).

- Work outcomes (eg, patient experience, safety, quality of care, individual and organisational performance).

13. Key findings that relate to the review questions.

The information extracted will then be summarised and tabulated in an Excel file. Each article will be assigned to two reviewers. The reviewers will work independently to extract the data; the data extracted by the pair of reviewers will be compared, and any discrepancies will be further discussed to ensure consistency between the reviewers. Conflicts will be discussed between the reviewers and consensus obtained. If there is difficulty in reaching a consensus, a third reviewer's opinion will be obtained. This process is undertaken so as to ensure accurate and reliable data collection.

\section{Stage 5: data summary and synthesis of results}

Quantitative methods using content analysis will be used to categorise study findings on factors associated with trust between patients, clients and healthcare providers. The collection of studies will be also examined for heterogeneity. Qualitative analysis on peer reviewed articles of qualitative interviews and focus group discussion will be conducted; it allows clear identification of themes arising from the data, facilitating prioritisation, higher order abstraction and theory development. The findings will be analysed (including descriptive numerical summary analysis and qualitative thematic analysis), discussed and reported.

In reviewing the instruments used to measure trust, they will be evaluated for validity and reliability, as well as to understand the domains which are measured, and how the domains are measured. 


\section{Stage 6: consultation}

A consultation exercise with stakeholders will be incorporated as a knowledge translation component of the scoping study methodology.

\section{Patient and public involvement}

Patients and public were not involved in the development of this scoping review protocol.

\section{Data management}

All data will be kept confidential and a master index of all studies reviewed will be maintained.

\section{DISCUSSION}

\section{Implications}

The findings will be discussed as they relate to the study purpose and implications for future research, practice and policy. The International Medical University will use the findings of this scoping review research to improve the understanding of trust in healthcare, in its endeavour to improve health services delivery by its faculty in its healthcare clinics and hospitals, and in its teaching and learning curriculum. The findings will also help faculty make evidence-based decisions to focus resources and research as well as help to advance the science in this area.

\section{Ethics and dissemination}

Ethical approval will be obtained for the research project from the Institutional Review Board of the International Medical University. Dissemination of the scoping review findings will be done through peer-reviewed publications, research reports and conference/seminar presentations.

Acknowledgements The team would like to acknowledge the following: (1) Mohammad Hisyamuddin (Librarian, International Medical University, Kuala Lumpur, Malaysia) who assisted in the initial search for the journal articles and developed the database of articles in the 'Ref works folder'. (2) Dr Teguh Haryo Sasongko Associate Professor Human Biology, School of Medicine International Medical University, Kuala Lumpur, Malaysia who assisted in the final review of the paper.

Contributors SR: main author, conceived the project, developed the conceptual framework for the study, analysed the preliminary data and wrote the manuscript, and also independent reviewer in the preliminary review. SJ: contributed in writing the manuscript and was one of the independent reviewers in the preliminary review. SY: contributed in performing the search working with the librarian to search for and compile the list of relevant journal articles and store in the specific 'Refworks' folder. GP: contributed in writing the manuscript. CPYK: contributed in writing the manuscript. SDA: contributed in writing the manuscript. All authors read and approved the final version of the manuscript.

Funding The authors have not declared a specific grant for this research from any funding agency in the public, commercial or not-for-profit sectors.

Competing interests None declared.

Patient consent for publication Not required.

Provenance and peer review Not commissioned; externally peer reviewed.

Open access This is an open access article distributed in accordance with the Creative Commons Attribution Non Commercial (CC BY-NC 4.0) license, which permits others to distribute, remix, adapt, build upon this work non-commercially, and license their derivative works on different terms, provided the original work is properly cited, appropriate credit is given, any changes made indicated, and the use is non-commercial. See: http://creativecommons.org/licenses/by-nc/4.0/.

ORCID iD

Supathiratheavy Rasiah http://orcid.org/0000-0002-3451-5024

\section{REFERENCES}

1 Calnan M, Rowe R. Trust matters in healthcare. In: Open university press. McGraw Hill Publication., 2008.

2 Pellegrini CA. Trust: the keystone of the patient-physician relationship. J Am Coll Surg 2017;224:95-102.

3 Gilson L. Trust and the development of health care as a social institution. Soc Sci Med 2003;56:1453-68.

4 Anderson LA, Dedrick RF. Development of the trust in physician scale: a measure to assess interpersonal trust in patient-physician relationships. Psychol Rep 1990;67:1091-100.

5 Caterinicchio RP. Testing plausible path models of interpersonal trust in patient-physician treatment relationships. Social Science \& Medicine. Part A: Medical Psychology \& Medical Sociology 1979;13:81-99.

6 Shore DA. Communicating in times of uncertainty: the need for trust. $J$ Health Commun 2003;8 Suppl 1:13-14.

7 Mechanic D, Meyer S. Concepts of trust among patients with serious illness. Soc Sci Med 2000;51:657-68.

8 Chipidza FE, Wallwork RS, Stern TA. Impact of the doctor-patient relationship. Prim Care Companion CNS Disord 2015;17.

9 Mechanic D. Public trust and initiatives for new health care partnerships. Milbank Q 1998;76:281-302.

10 Kohn LT, Corrigan JM, Donaldson MS. To err is human: building a safer health system. In: Institute of Medicine (US) Committee of quality of health care in America. National Academic Press (US), 2000.

11 Blendon RJ, Benson JM, Hero JO. Public trust in physicians - U.S. medicine in international perspective. N Engl J Med 2014;371:1570-2.

12 Arskey H, O'Malley L. Scoping studies: towards a methodological framework. Int J Soc Res Meth 2005;8:19-32.

13 Levac D, Colquhoun H, O'Brien KK. Scoping studies: advancing the methodology. Implementation Sci 2010;5. 